Special issue of the 3rd International Conference on Computational and Experimental Science and Engineering (ICCESEN 2016)

\title{
A New Computational Method \\ Based on Laguerre Polynomials for Solving Certain Nonlinear Partial Integro Differential Equations
}

\begin{abstract}
B. GÜRBÜZ, M. SEZER*
Manisa Celal Bayar University, Department of Mathematics, Faculty of Science and Letters, Manisa, Turkey

In this study, we consider some nonlinear partial integro-differential equations. Most of these equations are used as mathematical models in many problems of physics, biology, chemistry, engineering, and in other areas. Our main purpose is to propose a new numerical method based on the Laguerre and Taylor polynomials, called matrix collocation method, for the numerical solution of the mentioned nonlinear equations under the initial or boundary conditions. To show the effectiveness of this approach, some examples along with error estimations are illustrated by tables and figures.
\end{abstract}

DOI: 10.12693/APhysPolA.132.561

PACS/topics: nonlinear partial integro-differential equations, Laguerre polynomials, matrix and collocation methods, error estimations

\section{Introduction}

Nonlinear partial integro-differential equations frequently arise in many fields of engineering, mathematics, physics, finance, biology, and related areas [1-8]. There exists main challenges for solving these type of problems due to large range of variables, nonlinearity, and multidimensionality, and so on. Accordingly, the numerical methods are developed [9-16]. In this study, we present the Laguerre collocation method to solve certain nonlinear partial integro-differential equations in the form

$$
\begin{aligned}
& u_{t}(x, t)=g(x, t)+a(x, t) u_{x x}(x, t)+u^{2}(x, t) \\
& \quad+\int_{0}^{t} K(x, t, s) u(x, s) \mathrm{d} s
\end{aligned}
$$

under the initial and boundary conditions

$$
\begin{aligned}
& u(x, 0)=f(x) u_{t}(x, 0)=m(x), u(0, t)=h(t), \\
& 0 \leq x \leq l, 0 \leq t \leq T,
\end{aligned}
$$

where the functions $g, a, f, m$, and $h$ are continuous functions; and $K$ is the kernel function. Then we assume the approximate solution of the problem (1)-(2) in the truncated Laguerre series form

$$
\begin{aligned}
& u(x, t) \cong \sum_{n=0}^{N} \sum_{p=0}^{N} a_{n, p} L_{n, p}(x, t), \\
& L_{n, p}(x, t)=L_{n}(x) L_{p}(t) L_{n}(x)= \\
& \sum_{k=0}^{n} \frac{(-1)^{k}}{k !}\left(\begin{array}{l}
n \\
k
\end{array}\right) x^{k},
\end{aligned}
$$

where $n \in N, 0 \leq x \leq \infty$ and $a_{n, p}(n p=0,1, \ldots, N)$ are unknown Laguerre polynomial coefficients, and $N$ is any integer such that $N \geq 2[17,18]$.

*corresponding author; e-mail: burcu_grbz@yahoo.com

\section{Fundamental relations and Laguerre collocation method}

In this section, the approximate solution is presented. Initially, the matrix form of (3) is given

$$
\begin{aligned}
& {[u(x, t)]=\boldsymbol{L}(x) \overline{\boldsymbol{L}}(t) \boldsymbol{A} ;} \\
& \boldsymbol{L}(x)=\left[\begin{array}{lll}
\boldsymbol{L}_{0}(x) & \boldsymbol{L}_{1}(x) & \boldsymbol{L}_{N}(x)
\end{array}\right], \\
& \overline{\boldsymbol{L}}(t)=\operatorname{diag}(\boldsymbol{L}(t), \boldsymbol{L}(t), \ldots, \boldsymbol{L}(t)), \\
& \boldsymbol{A}=\left[\begin{array}{llll}
a_{i 0} & a_{i 1} & \ldots & a_{i N}
\end{array}\right]^{T},
\end{aligned}
$$

where $i=0,1, \ldots, N$, and we use the matrix relation

$\boldsymbol{L}(x)=\boldsymbol{X}(x) \boldsymbol{H}$ and $\overline{\boldsymbol{L}}(t)=\overline{\boldsymbol{X}}(t) \overline{\boldsymbol{H}} ;$

$$
\begin{aligned}
& \boldsymbol{X}(x)=\left[\begin{array}{llll}
1 & x & \ldots & x^{N}
\end{array}\right] ; \\
& \overline{\boldsymbol{X}}(t)=\operatorname{diag}(\boldsymbol{X}(t) \boldsymbol{X}(t), \ldots, \boldsymbol{X}(t)),
\end{aligned}
$$

$$
\boldsymbol{H}=
$$

$$
\begin{gathered}
{\left[\begin{array}{ccccc}
\frac{(-1)^{0}}{0 !}\left(\begin{array}{l}
0 \\
0
\end{array}\right) & \frac{(-1)^{0}}{0 !}\left(\begin{array}{l}
1 \\
0
\end{array}\right) & \frac{(-1)^{0}}{0 !}\left(\begin{array}{l}
2 \\
0
\end{array}\right) & \ldots & \frac{(-1)^{0}}{0 !}\left(\begin{array}{c}
N \\
0
\end{array}\right) \\
0 & \frac{(-1)^{1}}{1 !}\left(\begin{array}{l}
1 \\
1
\end{array}\right) & \frac{(-1)^{1}}{1 !}\left(\begin{array}{l}
2 \\
1
\end{array}\right) & \ldots & \frac{(-1)^{1}}{1 !}\left(\begin{array}{c}
N \\
1
\end{array}\right) \\
0 & 0 & \frac{(-1)^{2}}{2 !}\left(\begin{array}{l}
2 \\
2
\end{array}\right) & \ldots & \frac{(-1)^{2}}{2 !}\left(\begin{array}{c}
N \\
2
\end{array}\right) \\
\vdots & \vdots & \ddots & \vdots \\
0 & 0 & 0 & \ldots & \frac{(-1)^{N}}{N !}\left(\begin{array}{c}
N \\
N
\end{array}\right)
\end{array}\right],} \\
\overline{\boldsymbol{H}}=\operatorname{diag}(\boldsymbol{H}, \boldsymbol{H}, \ldots, \boldsymbol{H}) \\
{\left[u^{2}(x, t)\right]=\boldsymbol{L}(x, t) \overline{\boldsymbol{L}}(x, t) \overline{\boldsymbol{A}}=\boldsymbol{L}(x) \overline{\boldsymbol{L}}(t) \overline{\boldsymbol{L}}(x) \overline{\boldsymbol{L}}^{*}(t) \overline{\boldsymbol{A}}} \\
=\boldsymbol{X}(x) \boldsymbol{H} \overline{\boldsymbol{X}}(t) \overline{\boldsymbol{H} \boldsymbol{X}}(x) \overline{\boldsymbol{H}} \overline{\boldsymbol{X}^{*}}(t) \overline{\boldsymbol{H}^{*}} \overline{\boldsymbol{A}} ;
\end{gathered}
$$




$$
\overline{\bar{H}^{*}}=\operatorname{diag}(\overline{\boldsymbol{H}}, \overline{\boldsymbol{H}}, \ldots, \overline{\boldsymbol{H}}) \quad \overline{\boldsymbol{A}}=\left[\begin{array}{ccc}
\overline{\boldsymbol{A}}_{0} & \overline{\boldsymbol{A}}_{1} & \overline{\boldsymbol{A}}_{N}
\end{array}\right]^{T} .
$$

We also organize $\boldsymbol{X}(x), \overline{\boldsymbol{X}}(t)$ and their derivatives $\boldsymbol{X}^{\prime}(x)$, $\boldsymbol{X}^{\prime \prime}(x)$ and $\overline{\boldsymbol{X}}^{\prime}(t), \overline{\boldsymbol{X}}^{\prime \prime}(t)$ as

$$
\begin{aligned}
& \boldsymbol{X}^{\prime}(x)=\boldsymbol{X}(x) \boldsymbol{B}, \boldsymbol{X}^{\prime \prime}(x)=\boldsymbol{X}(x)(\boldsymbol{B})^{2}, \\
& \overline{\boldsymbol{X}}^{\prime}(t)=\overline{\boldsymbol{X}}(t) \overline{\boldsymbol{B}}, \overline{\boldsymbol{X}}^{\prime \prime}(t)=\overline{\boldsymbol{X}}(t)(\overline{\boldsymbol{B}})^{2} ; \\
& \boldsymbol{B}=\left[\begin{array}{ccccc}
0 & 1 & 0 & \ldots & 0 \\
0 & 0 & 2 & \ldots & 0 \\
\vdots & \vdots & \vdots & \ddots & \vdots \\
0 & 0 & 0 & N & 0 \\
0 & 0 & 0 & 0 & 0
\end{array}\right], \\
& \overline{\boldsymbol{B}}(\alpha, 0)=\operatorname{diag}(\boldsymbol{B}(\alpha, 0), \boldsymbol{B}(\alpha, 0), \ldots, \boldsymbol{B}(\alpha, 0)), \\
& \boldsymbol{B}(\alpha, 0)=\operatorname{diag}\left((\alpha)^{0},(\alpha)^{1}, \ldots,(\alpha)^{N}\right) .
\end{aligned}
$$

Then, the matrix relations of derivative forms of $u(x, t)$ is found with (4), (5), and (7):

$$
\begin{aligned}
& {\left[u_{x}(x, t)\right]=\boldsymbol{X}(x) \boldsymbol{B} \boldsymbol{H} \overline{\boldsymbol{L}}(t) \boldsymbol{A},} \\
& {\left[u_{x x}(x, t)\right]=\boldsymbol{X}(x) \boldsymbol{B}^{2} \boldsymbol{H} \overline{\boldsymbol{L}}(t) \boldsymbol{A},} \\
& {\left[u_{t}(x, t)\right]=\boldsymbol{X}(x) \boldsymbol{H} \overline{\boldsymbol{L}}^{\prime}(t) \boldsymbol{A} .}
\end{aligned}
$$

Besides, $K(x, t, s)$ can be expressed by the Taylor series as

$$
\begin{aligned}
& K(x, t, s)=\sum_{p=0}^{N} \sum_{q=0}^{N} \sum_{r=0}^{N} k_{p q r} x^{p} t^{q} s^{r}, \\
& k_{p q r}=\frac{1}{p ! q ! r !} \frac{\partial^{p+q+r} K(0,0,0)}{\partial x^{p} \partial t^{q} \partial s^{r}}, \\
& K=\left[k_{p q r}\right], p, q, r=0,1, \ldots, N
\end{aligned}
$$

and the matrix forms are

$$
\begin{gathered}
{[K(x, t, s)]=\boldsymbol{X}(x) \overline{\boldsymbol{X}}(t) \boldsymbol{K} \boldsymbol{X}^{T}(s),} \\
{[u(x, s)]=\boldsymbol{X}(x) \boldsymbol{H} \overline{\boldsymbol{X}}(s) \overline{\boldsymbol{H}} \boldsymbol{A}=} \\
\boldsymbol{X}(s) \boldsymbol{H} \overline{\boldsymbol{X}}(x) \overline{\boldsymbol{H}} \boldsymbol{A}^{*} .
\end{gathered}
$$

Then, the matrix form of integral part is obtained by (8):

$$
\begin{aligned}
& \int_{0}^{t} K(x, t, s) u(x, s) \mathrm{d} s= \\
& \quad \int_{0}^{t} \boldsymbol{X}(x) \overline{\boldsymbol{X}}(t) \boldsymbol{K} \boldsymbol{X}^{T}(s) \boldsymbol{X}(s) \boldsymbol{H} \overline{\boldsymbol{X}}(x) \overline{\boldsymbol{H}} \boldsymbol{A}^{*} \mathrm{~d} s= \\
& \quad \boldsymbol{X}(x) \overline{\boldsymbol{X}}(t) \boldsymbol{K} \boldsymbol{Q}(t) \boldsymbol{H} \overline{\boldsymbol{X}}(x) \overline{\boldsymbol{H}} \boldsymbol{A}^{*}, \\
& \boldsymbol{Q}(t)=\int_{0}^{t} \boldsymbol{X}^{T}(s) \boldsymbol{X}(s) \mathrm{d} s=\left[q_{m n}(t)\right] m, n=0,1,2 ., \\
& q_{m n}(t)=\frac{t^{m+n+1}}{m+n+1} \\
& \boldsymbol{A}_{i}^{*}=\left[a_{0 i} a_{1 i} \ldots a_{N i}\right]^{T}, \\
& \boldsymbol{A}^{*}=\left[\boldsymbol{A}_{0}^{*} \boldsymbol{A}_{1}^{*} \boldsymbol{A}_{N}^{*}\right]^{T} .
\end{aligned}
$$

By using $(6,8,10)$ we obtain the matrix form of $(1)$ :

$$
\begin{aligned}
\{ & \left.\boldsymbol{X}(x) \boldsymbol{H} \overline{\boldsymbol{L}}^{\prime}(t)-[a(x, t)] \boldsymbol{X}(x) \boldsymbol{B}^{2} \boldsymbol{H} \overline{\boldsymbol{L}}(t)\right\} \boldsymbol{A} \\
& -\left\{\boldsymbol{X}(x) \boldsymbol{H} \overline{\boldsymbol{X}}(t) \overline{\boldsymbol{H}} \boldsymbol{X}(x) \overline{\boldsymbol{H}}^{*}(t) \overline{\overline{\boldsymbol{H}}}^{*}\right\} \overline{\boldsymbol{A}} \\
& -\{\boldsymbol{X}(x) \overline{\boldsymbol{X}}(t) \boldsymbol{K} \boldsymbol{Q}(t) \boldsymbol{H} \overline{\boldsymbol{X}}(x) \overline{\boldsymbol{H}}\} \boldsymbol{A}^{*}=[g(x, t)]
\end{aligned}
$$

Correspondingly, we follow the same procedure for the conditions (2) by using (8):

$$
\begin{gathered}
{[u(x, 0)]=\boldsymbol{L}(x) \overline{\boldsymbol{L}}(0) \boldsymbol{A}=\boldsymbol{X}(x) \boldsymbol{H} \overline{\boldsymbol{L}}(0) \boldsymbol{A}=[f(x)]=\lambda,} \\
{\left[u_{t}(x, 0)\right]=\boldsymbol{L}(x) \overline{\boldsymbol{L}}^{\prime}(0) \boldsymbol{A}=\boldsymbol{X}(x) \boldsymbol{H} \overline{\boldsymbol{L}}^{\prime}(0) \boldsymbol{A}=[m(x)]=\mu,} \\
{[u(0, t)]=\boldsymbol{L}(0) \overline{\boldsymbol{L}}(t) \boldsymbol{A}=[h(t)]=\gamma .}
\end{gathered}
$$

By substituting the collocation points

$$
\begin{aligned}
x_{i} & =\frac{l}{N} i, i=0,1, \ldots, N, \\
t_{j} & =\frac{T}{N} j, j=0,1, \ldots, N, h=\frac{l}{N}
\end{aligned}
$$

into Eq. (11), we have the fundamental matrix equation

$$
\begin{aligned}
& \left\{\boldsymbol{X}\left(x_{i}\right) \boldsymbol{H} \overline{\boldsymbol{L}}^{\prime}\left(t_{j}\right)-\left[a\left(x_{i}, t_{j}\right)\right] \boldsymbol{X}\left(x_{i}\right) \boldsymbol{B}^{2} \boldsymbol{H} \overline{\boldsymbol{L}}\left(t_{j}\right)\right\} \boldsymbol{A} \\
& -\left\{\boldsymbol{X}\left(x_{i}\right) \boldsymbol{H} \overline{\boldsymbol{X}}\left(t_{j}\right) \overline{\boldsymbol{H}} \boldsymbol{X}\left(x_{i}\right) \overline{\boldsymbol{H}}^{*}\left(t_{j}\right) \overline{\overline{\boldsymbol{H}}}^{*}\right\} \overline{\boldsymbol{A}} \\
& -\left\{\boldsymbol{X}\left(x_{i}\right) \overline{\boldsymbol{X}}\left(t_{j}\right) \boldsymbol{K} \boldsymbol{Q}\left(t_{j}\right) \boldsymbol{H} \overline{\boldsymbol{X}}\left(x_{i}\right) \overline{\boldsymbol{H}}\right\} \boldsymbol{A}^{*} \\
& =\left[g\left(x_{i}, t_{j}\right)\right]=\boldsymbol{G} \\
& \boldsymbol{G}=\left[\begin{array}{llll}
\boldsymbol{G}_{1} & \boldsymbol{G}_{2} & \ldots & \boldsymbol{G}_{N}
\end{array}\right]^{T}, \boldsymbol{G}_{i}=\left[\begin{array}{llll}
g_{i 0} & g_{i 1} & \ldots & g_{i N}
\end{array}\right]^{T},
\end{aligned}
$$

or simply

$$
\begin{gathered}
\boldsymbol{W}\left(x_{i}, t_{j}\right) \boldsymbol{A}-\boldsymbol{M}\left(x_{i}, t_{j}\right) \overline{\boldsymbol{A}} \\
-\boldsymbol{W}^{*}\left(x_{i}, t_{j}\right) \boldsymbol{A}^{*}=\boldsymbol{G} .
\end{gathered}
$$

Similarly, we have the initial and boundary conditions (12) as

$$
\boldsymbol{U} \boldsymbol{A}=\lambda_{i}, \boldsymbol{V} \boldsymbol{A}=\mu_{i} \boldsymbol{Z} \boldsymbol{A}=\gamma_{j} .
$$

Subsequently, by replacing the row matrices (15) by the last rows of the augmented matrix (14), we have the required augmented matrix (14) as $\left[\tilde{\boldsymbol{W}} ; \tilde{\boldsymbol{M}} ; \tilde{\boldsymbol{W}}^{*} ; \tilde{\boldsymbol{G}}\right]$. By solving the system also by finding the Laguerre coefficients, the approximate solution $u(x, t)$ is obtained in (3) [19].

\section{Error estimation}

In this section, we give brief information about error estimation and we define the error function for the indicated points: $x=x_{\alpha}, t=t_{\beta} \in[l, 0] \times[0, T], \alpha, \beta=$ $0,1, \ldots$

$$
\begin{gathered}
E_{N}\left(x_{p} t_{q}\right)=\mid u_{t}\left(x_{p}, t_{q}\right)-g\left(x_{p}, t_{q}\right)\left(x_{p}, t_{q}\right) u\left(x_{p}, \alpha t_{q}\right) \\
-u^{2}\left(x_{p}, t_{q}\right)-\int_{0}^{t} K\left(x_{p}, t_{q}, s\right) u\left(x_{p}, s\right) \mathrm{d} s \mid \cong 0
\end{gathered}
$$

where $E_{N}\left(x_{p}, t_{q}\right) \leq 10^{-k_{\alpha \beta}}=10^{-k},(k>0)$ and the truncation limit $N$ is increased till the difference $E_{N}\left(x_{p}, t_{q}\right)$ at each of the points becomes less than $10^{-k}$. Also, we can use different error norms

$$
L_{2}-E_{N}\left(x_{\alpha}, t_{\beta}\right)=\sqrt{\sum_{i=1}^{n}\left(e_{i}\right)^{2}},
$$




$$
\begin{aligned}
& L_{\infty}-E_{N}\left(x_{\alpha}, t_{\beta}\right)=\max \left(e_{i}\right), 0 \leq i \leq n, \\
& \mathrm{RMS}-E_{N}\left(x_{\alpha}, t_{\beta}\right)=\sqrt{\sum_{i=1}^{n}\left(e_{i}\right)^{2} /(n+1)},
\end{aligned}
$$

where $e_{i}=u\left(x_{i}, \tau\right)-\hat{u}\left(x_{i}, \tau\right)$ where $e_{i}=u\left(x_{i}, \tau\right)-$ $\hat{u}\left(x_{i}, \tau\right)$; also $u$ and $\hat{u}$ are the exact and approximate solutions of the problem, respectively, and $\tau$ is an arbitrary time $t$ in $[0, T][20,21]$.

\section{Numerical experiments}

In this section, we show the accuracy of the method with respect to the numerical results which are calculated by Maple 18 and graphed in Matlab R2014b.

Example 5.1. We consider the parabolic partial integro-differential equation

$$
\begin{aligned}
& u_{t}(x, t)=g(x, t)+\frac{x^{2}(1-x)}{2} u_{x x}(x, t)+u^{2}(x, t) \\
& +\int_{0}^{t}(1-2 x) \mathrm{e}^{(s-t)} u(x, s) \mathrm{d} s
\end{aligned}
$$

with initial and boundary conditions $u(x, 0)=x(1-$ $x), u(0, t)=u(1, t)=, 0 \leq x t \leq 1$. The exact solution of the problem is $u(x, t)=x(1-x) \mathrm{e}^{-x t}$.

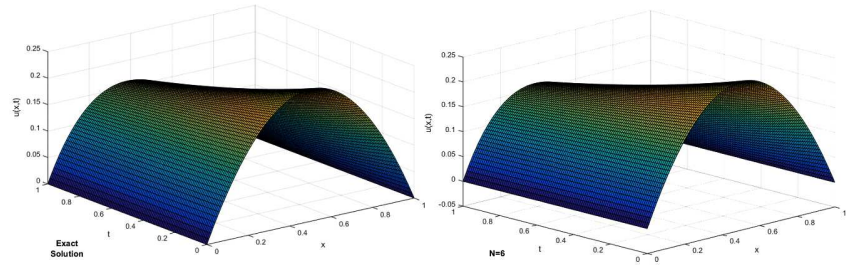

Fig. 1. Comparison of the numerical solutions for exact and $N=6$ solutions of example 5.1.

In Fig. 1 we can see the comparison between exact and $N=6$ solutions. In Table I the error differences are seen for the different $L$ norms.

Comparison of the errors of Example 5.1.

TABLE I for $N=3$ and $t=0.2$.

\begin{tabular}{c|c|c|c|c}
\hline \hline$x, t$ & Absolute & \multicolumn{3}{|c}{ Error analysis } \\
\cline { 3 - 5 } & error & $L_{2}$ & $L_{\infty}$ & RMS \\
\hline 0.0 & $1.8962 \mathrm{E}-04$ & $1.2361 \mathrm{E}-05$ & $3.0521 \mathrm{E}-06$ & $1.0200 \mathrm{E}-07$ \\
0.2 & $1.1598 \mathrm{E}-03$ & $3.5890 \mathrm{E}-04$ & $4.0251 \mathrm{E}-05$ & $2.1927 \mathrm{E}-06$ \\
0.4 & $3.1120 \mathrm{E}-04$ & $4.2532 \mathrm{E}-04$ & $1.0256 \mathrm{E}-04$ & $8.0241 \mathrm{E}-05$ \\
0.6 & $6.7519 \mathrm{E}-04$ & $2.0505 \mathrm{E}-03$ & $9.9523 \mathrm{E}-03$ & $3.3261 \mathrm{E}-04$ \\
0.8 & $4.2560 \mathrm{E}-03$ & $2.2520 \mathrm{E}-04$ & $2.0831 \mathrm{E}-05$ & $2.0237 \mathrm{E}-05$ \\
1.0 & $1.0027 \mathrm{E}-03$ & $4.0029 \mathrm{E}-03$ & $2.2391 \mathrm{E}-05$ & $3.9986 \mathrm{E}-05$
\end{tabular}

\section{Conclusion}

Nonlinear partial integro-differential equations play a key role on many different areas, recently. The certain nonlinear partial integro-differential equations are solved numerically with many methods $[22,23]$. In this respect, we have presented the Laguerre collocation method which has remarkable advantages such as fast computation time and reduced computational operations. Numerical results satisfied the applicability of the method. In conclusion, the capability of the method is confirmed and the presented method can also be extended to other type of models, although some modifications are required.

\section{Acknowledgments}

This work is founded by Manisa Celal Bayar University Department of Scientific Research Projects, with grant ref. 2014-151.

\section{References}

[1] M. Koru, O. Serçe, Acta Phys. Pol. A 130, 453 (2016).

[2] I. Akkurt, H.O. Tekin, A. Mesbahi, Acta Phys. Pol. A 128, 332 (2015).

[3] S. Fiori, T. Gong, H.K. Lee, Cogn. Comput. 7, 715 (2015).

[4] A.E. Tiryaki, R. Kozan, N.G. Adar, Acta Phys. Pol. A 128, B-344 (2015).

[5] N.M. Nagy, Acta Phys. Pol. A 128, B-260 (2015).

[6] A. Gepperth, C. Karaoguz, Cogn. Comput. 8, 924 (2016).

[7] M. Urbanek, D. Blaszkiewicz, Acta Phys. Pol. A 128, B-241 (2015).

[8] M.A. Correia, F.C. Mena, A.J. Soares, in: Dynamics, Games and Science, Vol. II, Eds. M.M. Peixoto, A.A. Pinto, D.A.J. Rand, Springer, Berlin 2011, p. 247.

[9] N. Zeng, Z. Wang, H. Zhang, F.E. Alsaadi, Cogn. Comput. 8, 143 (2016).

[10] M. Ozsoy, K. Pehlivan, M. Firat, N. Ozsoy, V. Ucar, Acta Phys. Pol. A 128, B-327 (2015).

[11] J. Yang, L. Gong, Y. Tang, J. Yan, H. He, L. Zhang, G. Li, Cogn. Comput. 7, 582 (2015).

[12] E. Boutalbi, L.A. Gougam, F. Mekideche-Chafa, Acta Phys. Pol. A 128, B-271 (2015).

[13] X. Nian, M. Sun, H. Guo, H. Wang, L. Dai, Cogn. Comput. 9, 225 (2017).

[14] A. Tari, M.Y. Rahimi, S. Sharmarad, F. Talati, J. Comput. Appl. Math. 228, 70 (2009).

[15] Z. Jachiewicz, B. Zubik-Kowal, Appl. Num. Meth. 56, 433 (2006).

[16] F. Fakhar-İzadi, M. Dehghan, Math. Meth. Appl. Sci. 36, 1485 (2013).

[17] B. Gürbüz, M. Sezer, C. Güler, J. Appl. Math. 2014, 682398 (2014).

[18] B. Gürbüz, M. Sezer, Acta Phys. Pol. A 130, 194 (2016).

[19] B. Gürbüz, M. Sezer, Biomath. Commun. 6, 1706047 (2016).

[20] B. Bülbül, M. Sezer, Math. Probl. Eng. 7, 869749 (2013).

[21] K. Zaibak, K. Bouakaz, Acta Phys. Pol. A 128, B330 (2015).

[22] Z. Avazzadeh, Z. Beygi Rizi, F.M. Maalek Ghaini, G.B. Loghmani, Eng. Anal. Bound. Elem. 36, 881 (2012).

[23] N. Bildik, A. Konuralp, Int. J. Nonlin. Sci. Numer. Simulat. 7, 65 (2006). 